\title{
Assessing the Correlation between Medical Students' Psychological Distress and Their Academic Performance in Makkah, Saudi Arabia
}

\author{
Abdulelah Nuqali*, Haneen Al Nazzawi, Shatha Felmban, Hatem Assiri, \\ Nisrine Felemban
}

Faculty of Medicine, Umm Al-Qura University, Makkah, Saudi Arabia

Email: *abdulelah.n@gmail.com

How to cite this paper: Nuqali, A., Al Nazzawi, H., Felmban, S., Assiri, H., \& Felemban, N. (2018). Assessing the Correlation between Medical Students' Psychological Distress and Their Academic Performance in Makkah, Saudi Arabia. Creative Education, 9, 1332-1341.

https://doi.org/10.4236/ce.2018.99099

Received: June 14, 2018

Accepted: July 15, 2018

Published: July 18, 2018

Copyright $\odot 2018$ by authors and Scientific Research Publishing Inc. This work is licensed under the Creative Commons Attribution International License (CC BY 4.0).

http://creativecommons.org/licenses/by/4.0/

\begin{abstract}
Background: Stresses associated with medical school expose students to psychological distress that can have negative consequences. The purpose of this study was to screen for psychological distress among medical students, identify stressors, and examine the correlation between these stressors and students' academic performance. Methods: This cross-sectional study was conducted with 219 medical students at the basic science and clinical clerkship training levels at Umm Al-Qura University in Makkah, Saudi Arabia, in 2014. The Mental Health Inventory (MHI-38) was used to assess students' psychological well-being and distress in terms of anxiety, depression, and life satisfaction. A questionnaire was also used to assess common stressors and collect demographic information and students' grade point average (GPA). Results: Male students had higher levels of depression, anxiety, and loss of control than female students did. The mean MHI-38 anxiety, depression, and life satisfaction scores were $34.69(S D=7.14), 15.11(S D=3.82)$, and $3.12(S D=$ 1.01) respectively, indicating the sample was slightly anxious, somewhat depressed, and somewhat satisfied with life. MHI-38 depression scores significantly $(p<0.05)$ predicted GPA: students with lower depression levels had a higher GPA. The distress level was equal among medical students in all academic years, and most reported experiencing academic-related stressors. Conclusions: Results showed that psychological distress is common among the sample but is most prominent among males. Less depressed students had a higher GPA, and academic-related stressors were most frequently reported by students. These findings highlight the need for addressing mental health issues and eliminating the risk factors.
\end{abstract}




\section{Keywords}

Medical Students, Emotional Problems, Psychological Tests, Anxiety, Depression

\section{Introduction}

Mental health is essential for the well-being of both individuals and communities. Psychological distress is a form of emotional distress that manifests as varying degrees of depression and anxiety and that may be associated with somatic symptoms like insomnia and fatigue (Keyes, 2002; Engen, 2008). Medical school is considered stressful due to the heavy workload and associated high level of stress, and medical students often possess certain personality qualities, such as perfectionism or performance-based self-esteem, that may increase their likelihood of developing mental health problems (Dahlin et al., 2011). During medical school, students are exposed to several stressors-whether related to academics, relationships, or transitional challenges-that threaten their mental health and may lead to negative consequences such as suicidal thoughts, impaired academic performance, and dropping out of school (Reisbig et al., 2012; Dyrbye et al., 2011; Jafari et al., 2012).

The most frequently presenting manifestations of psychological distress are burnout (49.6\%), stress (48.6\%), depression (46.5\%), fatigue (46.3\%), low mental quality of life (41.3\%), and low physical quality of life (22.3\%) (Dyrbye et al., 2011). Many studies have suggested that this type of distress manifests early, from the first year in medical school (Jafari et al., 2012; Simard \& Henry, 2009). Many studies have investigated the psychological distress spectrum (such as anxiety and depression) among medical students. However, in the present study, we sought to find the psychological well-being (such as general positive affect, emotional ties, and life satisfaction) and its effect on academic performance. Therefore, we had the following objectives: 1) to screen for psychological distress and its manifestations among medical students at Umm Al-Qura University; 2) to identify the stressors influencing psychological distress; and 3) to examine the correlation between these stressors and students' academic performance.

\section{Methods}

\subsection{Study Design, Participants, and Procedure}

This single-center, cross-sectional study was conducted at the Faculty of Medicine, Umm Al-Qura University, Makkah, Saudi Arabia, from March to June 2014. Umm Al-Qura University is a governmental, tuition-free institution where male students are separated from female students. After completing the premedical year, students compete for the available positions in the next year of medical school based on their overall grade point average (GPA). The following medical program curriculum is a 5-year program divided into two levels: 2 years 
of basic sciences and 3 years of clinical clerkships followed by a compulsory year of internship. In the 2013-2014 academic year, there were 1468 enrolled students, 750 of whom were female and 718 of whom were male. We used stratified random sampling to select the calculated necessary sample size of 288 students, which, based on previous studies (Ibrahim et al., 2013; El-Gilany et al., 2008), showed that the mid-range prevalence of psychological distress among medical students is around $25 \%$. To obtain a confidence level (power) of $95 \%$ and a confidence limit of $10 \%$, we aimed to study 288 students (calculated using the QuesGen Systems sample size calculator). Furthermore, we decided to approach 331 students because we expected that approximately $15 \%$ would either refuse to take part in the study or provide incomplete data. The sample size was stratified according to gender and academic year. Students were asked to complete a manual, self-administered bilingual (English and Arabic) questionnaire that we distributed at the university after regular lectures. The questionnaire measured psychological distress as well as demographic information, including gender, academic year, and GPA. In addition, we avoided administering the questionnaires at stressful times (e.g., during examinations) to avoid artificially inflated stress results.

\subsection{Measures}

To assess psychological distress, we used the Mental Health Inventory (MHI-38), which was developed by Veit and Ware (1983). It consists of 38 items that measure different aspects of psychological well-being experienced during the past 4 weeks, including the following: anxiety, depression, loss of behavioral/emotional control, general positive affect, emotional ties, and life satisfaction. We used a 6-point Likert-style scale, with responses ranging from 1 (high) to 6 (low); for example "extremely happy" to "dissatisfied" or "all of the time" to "none of the time". The MHI-38 was designed with no clinical cutoffs, and the psychological distress (negative emotion state) and psychological well-being (positive emotion state) global scales were complementary. Both global scales were scored such that higher scores indicate a higher level of each specific construct (Davies et al., 1998). The MHI-38 is a self-administrated questionnaire that takes about 10 minutes to complete. It has been studied thoroughly with large populations, and there is notable evidence for its validity. It reported good convergent and discriminant validity, and a Cronbach's alpha rating of 0.93 (DHHS, 2004). It also showed a high correlation with Multiple Sclerosis Quality of Life Inventory (MSQLI). The Arabic version of the MHI-38 was translated and validated in 2005 by the Queensland Transcultural Mental Health Centre (2014). We received approval to use this scale free of charge from RAND Health Corporation.

The questionnaire also comprised a scale to identify common stressors through a literature review of studies on human and veterinary medical students' mental health and through their clinical, administrative, and research experience (Reisbig et al., 2012). The scale aims to determine the relationships among the 
most common stressors and their impact on students' mental and general health and academic performance. It consists of 16 items divided into four categories: academic, family health, relationships, and transitional stressors. We used a 4-point Likert-type scale, with responses ranging from 0 (never) to 3 (always), with higher scores indicating greater stress levels.

The stressors were professionally translated by a native Arabic speaker who has a bachelor of arts degree in English. The Arabic version was then sent to a second professional translator who is also a native Arabic speaker and has a bachelor of arts degree in English and who back-translated the questionnaire into English to check the translated version's consistency. The back-translated version was nearly identical to the original English version, except for a few words that were subsequently corrected.

\subsection{Ethical Considerations}

We informed all the participants about the rationale for our study and its objectives. Information about the scales and how to answer the questionnaire was provided by trained data collectors (student coordinators in the male section and the investigators themselves in the female section). Students were informed that participation was optional and that their identities would remain anonymous. Awritten and signed voluntary informed consent was obtained at the time of the student's enrollment. All procedures were followed in accordance with the latest update of the Helinski declaration. The protocol was reviewed and approved by the Committee of Bio-Medical Ethics of the Faculty of Medicine, Umm Al-Qura University in Makkah, Saudi Arabia.

\subsection{Statistical Analysis}

The descriptive statistics comprised frequencies and percentages for categorical data and means and standard deviations for continuous data. A linear regression procedure was conducted to determine the predictors of GPA, with a $p$ value under 0.05 considered statistically significant. Non-directional hypotheses were reported, and only questionnaires with complete data were analyzed. The Statistical Package for the Social Sciences version 21 was used for data management and statistical analysis.

\subsubsection{Assessing Univariate Normality}

Histograms of the variables were examined. Since the distribution of GPA was non-normal, it was transformed using a natural log function (Tabachnick \& Fidell, 2007); the transformed variable had a normal distribution and was, thus, used in subsequent analysis procedures. The distributions of all MHI-38 subscales were normal, but those of the stressors were non-normal. Thus, all the stressor items were converted into binary variables; respondents who indicated that they did not experience the stressor were categorized into one group, while those who provided ratings from 1 to 3 were categorized into the second group. 


\subsubsection{Predictors of Academic Performance}

A hierarchical linear regression procedure was conducted to determine which of the MHI-38 subscales and stressors significantly predicted GPA, after controlling for gender and year at medical school. The assumptions of multivariate normality, linearity, and homoscedasticity were fulfilled, as the points clustered toward the diagonal in the normal probability plot and the plot of the studentized deleted residuals by the standardized predicted values yielded a random scatter (Norušis, 1991).

\section{Results}

\subsection{Preliminary Screening Procedures}

\subsubsection{Checking for Missing Values}

Ninety-eight questionnaires had missing values and were not included in the subsequent data analyses. The group of respondents with missing values did not differ significantly from the group of respondents without missing values in terms of year at medical school $(\mathrm{p}=0.304)$, GPA $(\mathrm{p}=0.438)$, academic stressors $(\mathrm{p}=0.938)$, non-academic stressors $(\mathrm{p}=0.909)$, MHI-38 positive affect scores ( $\mathrm{p}$ $=0.274)$, or MHI-38 emotional ties scores $(p=0.973)$. However, there was a significant difference between the groups in terms of gender ( $p=0.001) ; 41 \%$ of the females, compared to $21.2 \%$ of the males, had missing values. In addition, respondents with missing values had higher MHI-38 life satisfaction $(\mathrm{p}=0.012)$, lower MHI-38 anxiety $(\mathrm{p}=0.002)$, lower MHI-38 depression $(\mathrm{p}=0.004)$, and lower MHI-38 behavioral/emotional control loss scores $(\mathrm{p}=0.008)$ than respondents without missing values did.

\subsection{Descriptive Statistics}

\subsubsection{Description of the Sample}

Out of 331 medical students invited to take part in the study, 219 agreed to participate (response rate $=66 \%$ ). There were slightly more males $(54.3 \%)$ than females. Sixth-year medical students formed the largest group (22.8\%), and fifth-year students the smallest (16.0\%).

\subsubsection{Descriptive Statistics for the MHI-38 Subscales}

\section{1) Differences between males and females}

The findings in Table 1 reveal that males had significantly higher MHI-38 psychological distress, anxiety, depression, and loss of control scores than females did. However, there were no significant gender differences in terms of GPA, psychological well-being, positive affect, emotional ties, and life satisfaction.

\section{2) Differences across academic years}

Only GPA differed significantly across academic years, F $(4,214)=31.94, \mathrm{p}<$ 0.001. Post-hoc Tukey comparisons revealed that second-year students had a significantly higher GPA than third-, fourth-, fifth-, and sixth-year students did (ps $<0.001$ ). Third-year students had significantly higher GPA than fifth- and 
Table 1. Means, Standard Deviations, and Independent t-Test Results for the Study Variables by Gender $(\mathrm{N}=219)$.

\begin{tabular}{ccccccccc}
\hline & $\begin{array}{c}\text { Score } \\
\text { range }\end{array}$ & $\begin{array}{c}\text { Study } \\
\text { range }\end{array}$ & \multicolumn{2}{c}{ Males } & \multicolumn{2}{c}{ Females } \\
\hline Variable & & & $M$ & $S D$ & $M$ & $S D$ & $t$ & Sig. \\
\hline Grade point average & - & $1-4$ & 3.09 & 0.57 & 3.23 & 0.57 & -1.79 & 0.075 \\
Mental Health Index & $38-226$ & $81-210$ & 153.81 & 25.39 & 146.24 & 26.99 & 2.13 & 0.034 \\
Psychological distress & $24-142$ & $53-135$ & 100.03 & 16.51 & 94.06 & 17.37 & 2.60 & 0.010 \\
Anxiety & $9-54$ & $18-51$ & 35.69 & 7.19 & 33.51 & 6.93 & 2.27 & 0.024 \\
Depression & $4-23$ & $5-22$ & 15.59 & 3.69 & 14.53 & 3.91 & 2.06 & 0.041 \\
$\begin{array}{c}\text { Loss of control } \\
\text { Psychological }\end{array}$ & $9-53$ & $16-52$ & 41.50 & 5.74 & 38.97 & 6.39 & 3.08 & 0.002 \\
well-being & $14-84$ & $17-76$ & 44.23 & 11.55 & 45.82 & 11.99 & -1 & 0.319 \\
Positive affect & $10-60$ & $12-56$ & 32.06 & 8.59 & 33.56 & 8.81 & -1.27 & 0.204 \\
Emotional ties & $2-12$ & $2-12$ & 5.95 & 2.42 & 5.75 & 2.43 & 0.61 & 0.545 \\
Life satisfaction & $1-6$ & $1-6$ & 3.07 & 0.94 & 3.19 & 1.09 & -0.90 & 0.371 \\
\hline
\end{tabular}

sixth-year students did (ps $<0.001$ ). Fourth-year students had a significantly higher GPA than fifth- and sixth-year students $\operatorname{did}(\mathrm{ps}<0.001)$.

\subsubsection{Descriptive Statistics for the Stressors}

Table 2 shows the list of stressors and the number of students who indicated that they experienced each one sometimes, often, or always. Most of the students reported experiencing academic-related stressors that include, unable to complete studies in allotted time, workload too heavy and concerned about academic performance. Family and relationship stressors were the least reported by students.

\subsection{Predictors of Academic Performance}

The findings reveal that, after controlling for gender and year at medical school, only MHI-38 depression scores significantly predicted GPA ( $p<0.05)$. The less depressed the students were, the higher was their GPA.

\section{Discussion}

In this study, we screened for psychological distress among medical students by assessing the most common manifestations of psychological distress, including anxiety, depression, loss of behavioral or emotional control, positive affect, emotional ties, and life satisfaction. Overall, the respondents were relatively healthy and had average psychological well-being scores. The estimated prevalence of psychological distress has been found to vary among medical students in different countries, which may be explained by differences in background cultures of each sample and in the instruments used. In Saudi Arabia, El-Gilany et al. (2008) reported a stress rate of $28.9 \%$ among Saudi medical students, whereas 
Table 2. Frequencies and percentages of students reporting specific stressors $(\mathrm{N}=219)$.

\begin{tabular}{ccc}
\hline Variable & Frequency & Percentage \\
\hline Being behind in studies & 199 & 90.9 \\
Concerned about academic performance & 207 & 94.5 \\
Workload too heavy & 204 & 93.2 \\
Unable to complete studies in allotted time & 213 & 97.3 \\
Difficulty understanding textbook/lectures & 174 & 79.5 \\
Uncertain of school expectations & 184 & 84 \\
Less intelligent than other students & 108 & 49.3 \\
Transitioning to new area & 67 & 30.6 \\
Homesickness & 80 & 36.5 \\
Don't fit in with other students & 103 & 47 \\
Transportation difficulties & 59 & 26.9 \\
Financial concerns & 81 & 37 \\
Family illness & 53 & 24.2 \\
Death of a loved one & 48 & 21.9 \\
Balancing school with romantic life & 163 & 74.4 \\
Relationship conflicts with partner/spouse & 48 & 21.9 \\
\hline
\end{tabular}

Abdulghani et al. (2011) found the prevalence of $63.8 \%$ among medical students in another university.

When we assessed manifestations of psychological distress, we found that the students were slightly anxious and somewhat depressed. Generally, these findings are consistent with those of other studies conducted in the same region and worldwide, which revealed the higher prevalence of anxiety and depression among medical students in comparison to the general population (Dyrbye et al., 2006). These findings could be explained by either the presence of real factors such as a new and highly competitive environment or the heavy workload that negatively affects mental health or the medical students who had a high level of awareness of depression and anxiety symptoms.

Loss of behavioral or emotional control is another manifestation of psychological distress. In the present study, students were found to have moderate control of their behaviors and emotions. Further, male students reported higher levels of depression, anxiety, and loss of control than female students did, but there were no significant differences in terms of GPA, positive affect, emotional ties, or life satisfaction. This finding is similar to previous findings; for example, Amr et al. (2013) conducted a study in Saudi Arabia that revealed that male students were significantly more likely to suffer from depression and anxiety than female students. However, this result contrasts with other previous studies; for example, Abdulghani et al. (2011) reported higher stress scores for female (75.7\%) than male (57\%) students, which is consistent with the systemic review 
study results of Dyrbye et al. (2006), who reviewed 40 articles on medical student psychological distress and concluded that "overall, the studies suggest psychological distress may be higher among female students".

Other previous studies revealed no difference between male and female students in terms of depression and anxiety (Dyrbye et al., 2006). A possible explanation for the gender differences found in our study is the sociocultural factors. The present study showed no relationship between academic year and psychological distress manifestations of depression, loss of behavioral control, and life satisfaction, which is not consistent with the findings of previous studies. In Iranian (Jafari et al., 2012) and Swedish (Dahlin et al., 2011) studies, medical students in the basic science years experienced a higher level of distress, whereas a study from Pakistan (Shah et al., 2010) reported a higher degree of distress among fourth-and final-year students. It may be that our finding is inconsistent with the abovementioned results because of the different tools used to assess psychological distress or variation in the curricula across these universities and countries. Thus, we cannot conclude that psychological distress progresses, regresses, or stays the same throughout medical school. Consequently, a longitudinal study is recommended to assess changes in the course of psychological distress throughout medical school. This would help to address the fact that little research has been carried out to investigate the consequences of depression and anxiety among medical students in Saudi Arabia, especially in terms of the negative impact on academic performance.

The present study did not find any association between anxiety and academic performance, which contrasts with the findings of most previous studies (Dyrbye et al., 2005). In addition, students who experienced academic-related stressors reported being unable to study in the allotted time as the top cause, followed by concern about academic performance and workload being too heavy.

Aboalshamat et al. (2015) assessed psychological well-being of preclinical (second and third years) dental and medical students at Umm Al-Qura University in Makkah, Saudi Arabia. They showed that there is a significant relationship between depression and students' academic performance, which is consistent with our results. However, in contrast to our findings, female medical students had a higher psychological distress level compared to male students. This difference might be explained by the different scales used to assess psychological distress. Further, we assessed psychological distress in both preclinical (second and third years) and clinical (fourth, fifth, and sixth years) medical students, and their results are limited to preclinical medical students.

In conclusion, psychological distress is common among medical students, and depression, specifically, might affect their academic performance. Academic-related stressors were the most frequently reported type. These findings highlight the need for increasing awareness of students' psychological distress for policymakers, university course administrators, and parents, all of whom could contribute to establishing screening programs, effective psychological services, and possible curriculum modifications to prevent further negative consequences. 


\section{Limitations}

We used the MHI-38 to gain a better understanding of psychological distress as a global measure. However, there are no well-established cutoff meaningful values for this scale, which makes interpreting the results more challenging.

This cross-sectional study reflects the experiences of medical students at Umm Al-Qura University; therefore, the generalizability of the study results is limited. We recommend that similar studies be conducted at other universities with different programs and curricular models. A study conducted at one UK medical school among medical students indicated the prevalence and persistence of depression and anxiety and showed that longitudinal studies are helpful. We recommend that longitudinal studies be conducted to detect early manifestations of psychological distress, whether transient or repeated. An additional limitation was the lack of data collection on factors that could be related to depression, anxiety, and academic performance. These include absenteeism, socioeconomic status, behavioral factors such as frequency of smoking and exercise, presence of close friendships, and religious beliefs. Another limitation was that GPA was self-reported in this study, and some students-especially females-refused to report this. Fear of being envied (due to cultural beliefs), underestimated, or identified are the possible causes for this reluctance. Respondents with missing values did not differ significantly from respondents without missing values; thus, truthfulness is a concern in self-reported GPAs.

\section{Acknowledgements}

We thank Dr. Victoria Chiongbian for assistance with statistical analysis, and Norah Al Faadhel and Nora Al Omair for assistance with translation.

\section{References}

Abdulghani, H. M., AlKanhal, A. A., Mahmoud, E. S., Ponnamperuma, G. G., \& Alfaris, E. A. (2011). Stress and Its Effects on Medical Students: A Cross-Sectional Study at a College of Medicine in Saudi Arabia. Journal of Health, Population and Nutrition, 29, 516-522. https://doi.org/10.3329/jhpn.v29i5.8906

Aboalshamat, K., Hou, X. Y., \& Strodl, E. (2015). Psychological Well-Being Status among Medical and Dental Students in Makkah, Saudi Arabia: A Cross-Sectional Study. Medical Teacher, 37, S75-S81. https://doi.org/10.3109/0142159X.2015.1006612

Amr, M., Amin, T. T., Sahoo, S., Al Malki, S., Al Samail, M., Al Qahtani, N. et al. (2013). Depression and Anxiety among Saudi University Students: Prevalence and Correlates. The Arab Journal of Psychiatry, 24, 1-7. https://doi.org/10.12816/0000092

Dahlin, M., Nilsson, C., Stotzer, E., \& Runeson, B. (2011). Mental Distress, Alcohol Use and Help-Seeking among Medical and Business Students: A Cross-Sectional Comparative Study. BMC Medical Education, 11, 92.

Davies, A. R., Sherbourne, C. D., Peterson, J. R., \& Ware, J. E. (1998). Scoring Manual: Adult Health Status and Patient Satisfaction Measures Used in RAND's Health Insurance Experiment. Santa Monica, CA: RAND.

DHHS (2004). Community Integration for Older Adults with Mental Illnesses: Overcoming Barriers and Seizing Opportunities. DHHS Pub. No. (SMA) 05-4018, Rockville, 
MD: Center for Mental Health Services, Substance Abuse and Mental Health Services Administration.

Dyrbye, L. N., Harper, W., Durning, S. J., Moutier, C., Thomas, M. R., Massie, F. S. Jr. et al. (2011). Patterns of Distress in US Medical Students. Medical Teacher, 33, 834-839. https://doi.org/10.3109/0142159X.2010.531158

Dyrbye, L. N., Thomas, M. R., \& Shanafelt, T. D. (2005). Medical Student Distress: Causes, Consequences, and Proposed Solutions. Mayo Clinic Proceedings, 80, 1613-1622. https://doi.org/10.4065/80.12.1613

Dyrbye, L. N., Thomas, M. R., \& Shanafelt, T. D. (2006). Systematic Review of Depression, Anxiety, and Other Indicators of Psychological Distress among U.S. and Canadian Medical Students. Academic Medicine, 81, 354-373. https://doi.org/10.1097/00001888-200604000-00009

El-Gilany, A. H., Amr, M., \& Hammad, S. (2008). Perceived Stress among Male Medical Students in Egypt and Saudi Arabia: Effect of Sociodemographic Factors. Annals of Saudi Medicine, 28, 7.

Engen, G. (2008). Psychosocial Predictors of Psychological Distress and Life Satisfaction in Norwegian University Students. University of Oslo: Faculty of Medicine, Department of General Practice and Community Medicine.

Ibrahim, N., Al-Kharboush, D., El-Khatib, L., Al-Habib, A., \& Asali, D. (2013). Prevalence and Predictors of Anxiety and Depression among Female Medical Students in King Abdulaziz University, Jeddah, Saudi Arabia. Iranian Journal of Public Health, 42, 726-736.

Jafari, N., Loghmani, A., \& Montazeri, A. (2012). Mental Health of Medical Students in Different Levels of Training. International Journal of Preventive Medicine, 3, 107-122.

Keyes, C. L. M. (2002). Selecting Outcomes for the Sociology of Mental Health: Issues of Measurement and Dimensionality. Journal of Health and Social Behavior, 43, 207-222. https://doi.org/10.2307/3090197

Norušis, M. J. (1991). SPSS Base Systems User's Guide. Englewood Cliffs, NJ: Prentice-Hall.

Queensland Transcultural Mental Health Centre (2014). Translation and Cultural Contextualisation of the Mental Health Inventory into 13 Community Languages.

Reisbig, A. M. J., Danielson, J. A., Wu, T. F., McArthur, H. Jr., Krienert, A., Girard, D. et al. (2012). A Study of Depression and Anxiety, General Health, and Academic Performance in Three Cohorts of Veterinary Medical Students across the First Three Semesters of Veterinary School. Journal of Veterinary Medical Education, 39, 341-358. https://doi.org/10.3138/jvme.0712-065R

Shah, M., Hasan, S., Malik, S., \& Sreeramareddy, C. T. (2010). Perceived Stress, Sources and Severity of Stress among Medical Undergraduates in a Pakistani Medical School. BMC Medical Education, 10, 2. https://doi.org/10.1186/1472-6920-10-2

Simard, A. A., \& Henry, M. (2009). Impact of a Short Yoga Intervention on Medical Students' Health: A Pilot Study. Medical Teacher, 31, 950-952. https://doi.org/10.3109/01421590902874063

Tabachnick, B. G., \& Fidell, L. S. (2007). Using Multivariate Statistics (5th ed.). San Francisco, CA: Pearson. 